\title{
BMJ Open Public perceptions of changing the terminology for low-risk thyroid cancer: a qualitative focus group study
}

\author{
Brooke Nickel, ${ }^{\oplus 1,2}$ Caitlin Semsarian, ${ }^{3}$ Ray Moynihan,, ${ }^{1,4}$ Alexandra Barratt, ${ }^{1}$ \\ Susan Jordan, ${ }^{5,6}$ Donald McLeod, ${ }^{5,7}$ Juan P Brito, ${ }^{8}$ Kirsten McCaffery ${ }^{1,2}$
}

To cite: Nickel B, Semsarian C, Moynihan $\mathrm{R}$, et al. Public perceptions of changing the terminology for low-risk thyroid cancer: a qualitative focus group study. BMJ Open 2019;9:e025820. doi:10.1136/ bmjopen-2018-025820

- Prepublication history and additional material for this paper are available online. To view these files, please visit the journal online (http://dx.doi. org/10.1136/bmjopen-2018025820).

Received 6 August 2018

Revised 18 December 2018

Accepted 19 December 2018

Check for updates

(C) Author(s) (or their employer(s)) 2019. Re-use permitted under CC BY-NC. No commercial re-use. See rights and permissions. Published by BMJ.

For numbered affiliations see end of article.

Correspondence to

Brooke Nickel;

brooke.nickel@sydney.edu.au

\section{ABSTRACT}

Objectives To investigate public perceptions of overdiagnosis and overtreatment in low-risk thyroid cancer and explore opinions regarding the proposed strategy to change the terminology of low-risk cancers.

Design Qualitative study using focus groups that included a guided group discussion and presentation explaining thyroid cancer, overdiagnosis and overtreatment, and proposed communication strategies. Transcripts were analysed thematically.

Setting Sydney, Australia.

Participants Forty-seven men and women of various ages from a range of socioeconomic backgrounds with no personal history of thyroid cancer.

Results Participants had low pre-existing general awareness of concepts of overdiagnosis and overtreatment and expressed concern regarding this new information in relation to thyroid cancer. Overall, participants understood why the strategy to change the terminology was being proposed and could see potential benefits including reducing the negative psychological impact and stigma associated with the term 'cancer'; however, many still had reservations about the strategy. The majority of the concerns were around their worry about the risk of further disease progression and that changing the terminology may create confusion and cause patients not to take the diagnosis and its associated managements seriously. Despite varied views towards the proposed strategy, there was a strong overarching desire for greater patient and public education around overdiagnosis and overtreatment in both thyroid cancer and cancer generally in order to complement any revised terminology and/or other mitigation strategies.

Conclusions We found a strong and apparently widely held desire for more information surrounding the topic of overdiagnosis and overtreatment. Careful consideration of how to inform both the public and current patients about the implications of a change in terminology, including changes to patients' follow-up or treatments, would be needed if such a change were to go ahead.

\section{INTRODUCTION}

In recent years, the incidence of thyroid cancer has dramatically increased worldwide, driven largely by the detection of papillary thyroid cancers (PTCs) ${ }^{1}$ which are typically small and slow-growing. Autopsy studies

\section{Strengths and limitations of this study}

- First study to investigate public perceptions of information about thyroid cancer overdiagnosis and overtreatment and responses to the possibility of changing the terminology of low-risk cancers.

- Participants varied in age, gender and socioeconomic status and were recruited using random digit dialling by an independent professional recruitment company.

- Information provided to participants was developed by a multidisciplinary team, including a consumer representative, and was reviewed by two independent thyroid cancer clinicians.

- Due to the amount and complexity of information, participants may not have comprehensively understood all concepts presented.

conducted on people who died of causes unrelated to the thyroid revealed latent PTCs in more than $10 \%$ of the general population. ${ }^{2}$ The existence of this reservoir of indolent disease, increasing use of clinical imaging and more intensive pathological scrutiny of thyroid resection specimens has led to increased incidental detection and diagnosis of small PTCs. ${ }^{3}$ Although rates vary, approximately half of new cases of PTC have been reported to be $<1 \mathrm{~cm}$ in diameter (known as papillary thyroid microcarcinomas) ${ }^{4-6}$

Most patients with PTC are treated with thyroid surgery (either total thyroidectomy or hemithyroidectomy). However, Japanese studies have shown that in patients with subcentimetre PTCs (low-risk PTCs), active surveillance leads to similar disease outcomes (growth, metastasis and mortality) compared with those who undergo immediate surgery. ${ }^{7}$ The potential for primary active surveillance of low-risk PTCs is significant as thyroid surgery may cause complications, including the risk of voice changes and issues with maintain normal calcium levels. ${ }^{8}$ Furthermore, lifelong reliance on daily thyroid hormone replacement medication is required for 
all thyroidectomy patients and a small proportion of hemithyroidectomy patients with its own burden of treatment. ${ }^{9}$ Although its use has been shown to be limited in clinical practice, ${ }^{10}$ the option of active surveillance for low-risk PTCs is beginning to be acknowledged in clinical practice guidelines. ${ }^{11}$

This rise of thyroid cancer incidence and the preferred used of thyroid surgery as a treatment option for low-risk PTC has raised concerns about thyroid cancer overdiagnosis and overtreatment. ${ }^{12}$ Recently, changing the terminology used to describe low-risk conditions has been recommended as a possible solution, to help prevent overdiagnosis and the associated harms of overtreatment. ${ }^{3}{ }^{13} 14$ Studies have previously shown how different terminologies affect treatment preferences for non-invasive breast cancer (ductal carcinoma in situ; DCIS) ${ }^{1516}$ and more recently similar studies have demonstrated similar findings for low-risk PTC. ${ }^{17}$ Removing the term 'cancer' in the description of both of these conditions led to higher preferences for non-surgical treatment in research settings. A recent systematic review, which includes the studies on DCIS, supports these findings and shows that the terminology used to describe various conditions can impact people's decisions about treatment as well as their psychological responses. ${ }^{18}$ Additionally, reference to 'cancer' was recently removed from a subtype of PTC ('non-invasive follicular thyroid neoplasm with papillary-like nuclear features'), which is identified after a total or hemithyroidectomy, to eliminate the need for ongoing follow-up and reduce potential patient anxiety. ${ }^{19}$ Taken together, this evidence indicates that removing 'cancer' from the terms used to describe of low-risk conditions, including low-risk PTC, may ameliorate adverse psychological outcomes, reduce overtreatment and is achievable in real world practice. However, to our knowledge there has as yet been no investigation of community perceptions of this strategy.

In this study, we aimed to investigate community perceptions of overdiagnosis and overtreatment in PTC, and the proposal to change the terminology of low-risk PTC to help inform mitigation strategies for overdiagnosis and overtreatment in this and other low-risk cancers.

\section{METHODS}

\section{Study design}

We conducted a qualitative focus group study to investigate what the community thought and felt about thyroid cancer overdiagnosis and overtreatment communication. The qualitative design involving focus groups with face-to-face contact with participants helped enable us to communicate potentially counterintuitive information about thyroid cancer and overdiagnosis ${ }^{20}$ effectively.

\section{Participant recruitment}

We recruited a community sample of men and women in Sydney, Australia aged $\geq 18$ years with no previous diagnosis of thyroid cancer to ensure that participants would not be biased by previous experience of diagnosis and treatment. An independent research recruitment organisation (Taverner Research) used random digit dialling to approach potential participants. Trained Taverner interviewers used a brief series of questions to determine participant eligibility, with participants not fluent in English and those with a personal diagnosis of thyroid cancer excluded. To gain a diverse range of perspectives, we used quota sampling to ensure inclusion of participants with varying levels of education. Those who agreed to participate were emailed a participant information statement and consent form. Each participant was given a \$100 gift voucher as a thank you for their attendance.

We aimed to conduct six focus groups with approximately eight participants in each in order to achieve thematic saturation as indicated by data redundancy, ${ }^{21} 22$ that is, when participants no longer raised original themes.

\section{Patient and public involvement}

Aconsumer representative (from Health Care Consumers' Association) was involved in the development and review of study materials. Study participants were a community sample recruited from the general Australian public.

\section{Focus group presentation and discussion}

The focus groups were conducted in February 2018. Sessions were convened at three locations that were convenient to the participants. Each session comprised: introduction and consent, demographic questionnaire, warm-up discussion, a detailed presentation and a final questionnaire. The presentation was divided into sections and interspersed with guided discussions about the content. Sessions lasted approximately 2 hours and were facilitated by two female moderators (BN and CS). Both moderators had experience and/or were trained in conducting qualitative focus groups and interviews.

At each focus group, BN gave an audiovisual presentation (see online supplementary appendix 1 for slides) that had been developed and reviewed by a multidisciplinary team of public health researchers with an interest in reducing overdiagnosis and overtreatment, health psychologists, clinicians, thyroid cancer experts and a consumer representative. Two independent thyroid cancer clinicians also reviewed the presentation before the focus groups commenced. The presentation included a combination of pictures, plain language and infographics appropriate for a range of numeracy levels. ${ }^{23} \mathrm{We}$ presented a range of published evidence showing overdiagnosis $^{324}$ and overtreatment ${ }^{7}$ of thyroid cancer using simple pictograms, and annotated graphs. The presentation was piloted with three different groups resulting in minor refinement.

In the group discussions, participants shared their responses to the information presented and how they felt about overdiagnosis and overtreatment in thyroid cancer and the proposed strategy of changing low-risk cancer terminology. Box 1 summarises the presentation content 
Box 1 Focus group presentation topics and key discussion questions

- Introduction to thyroid cancer (nature, detection and treatment).

- Introduction to cancer overdiagnosis, with graph to illustrate the varying growth rates of cancers and efficacy of screening.

- Introduction to overtreatment.

- Evidence of thyroid cancer overdiagnosis and overtreatment, various studies explained with statistical visuals:

- Autopsy studies showing latent thyroid cancers in $10 \%$ of the general population, ${ }^{2}$ with shaded pictogram.

- Dramatic increase in South Korean thyroid cancer incidence with little change in mortality rate, ${ }^{24}$ with annotated graph.

- Japanese studies showing similar outcomes in disease progression and death between patients who chose to monitor their small papillary thyroid cancers with scans and those who chose immediate surgery. ${ }^{7}$

- How do you feel about overdiagnosis and overtreatment of thyroid cancer?

- Introduction to proposed strategy of changing the terminology used to describe low-risk cancers, with graphic showing increased power of technology to detect cancer and excerpts of recommendations from reputable journals:

- Overall, how do you feel about this proposed strategy-to change the terminology of low-risk cancers including papillary thyroid cancer?

- What do you think are the pros and cons of this strategy? With responses tabulated on a whiteboard.

- Evidence of changing terminology, with examples of changed terminologies:

- If some sort of change to the terminology of low-risk thyroid cancers were to be made, what do you think would be the best term to use?

- Some terms have already been proposed or tested. These are 'abnormal cells', 'Iesion' and 'neoplasm/neoplasia'. How do you feel about these terms?

- Other possible descriptions, with the following words arranged on screen: 'cyst', 'lump', 'indolent lesion', 'precancer', 'benign', 'nodule', 'pre-invasive', 'common growth', 'standard tumour', 'preliminary mass', 'others':

- Which terms do you think best explain these types of conditions? - Do you have any ideas about other possible terms?

and gives an example of the types of questions asked to guide the discussions. At the beginning of each group we made it clear that some information presented may be unfamiliar, or cause disagreements among the group. We highlighted that we wanted to hear their honest thoughts and opinions and emphasised our neutrality to participant's opinions throughout by repeating statements of this kind, and by not interpreting views expressed by participants as positive or negative. A proposed strategy for thyroid cancer communication was able to be debated for and against, and these contrasting opinions led participants to articulate their own points of view in greater depth. ${ }^{25}$ Throughout the presentation, we strongly encouraged participants to request clarification as often as required.

\section{Data collection and analysis}

Focus group discussions were audio recorded, transcribed verbatim and analysed to identify recurring themes and data patterns. The data was managed using NVivo V.11 software. The analysis initially took a data-driven approach to ensure that the findings were grounded in participant's responses. Two researchers (BN and CS) independently reviewed transcripts in NVivo and developed codes in an iterative process. This approach, along with the research questions that shaped the presentation and the notes taken by researchers (BN and CS) during and following each focus group, formed the basis of the initial coding framework. Using constant comparison, ${ }^{21} 26$ the two researchers continually looked for similarities and differences in the data and in coding within and across transcripts. By comparing and discussing each aspect of the data a final coding framework developed. Final coding was performed by the two researchers in NVivo, with BN coding all of the data and CS double coding half. Once coded, the data was examined to identify overarching themes and concepts. Throughout this process, the two researchers met regularly with qualitative expert KM, and the coding framework and data interpretation were discussed and agreed on with members of the research team.

We also administered brief written questionnaires at the start and end of each focus group, which included questions about demographics (multiple choice responses), reactions and opinions regarding overdiagnosis (5-point Likert scale and yes/no responses), overtreatment and terminology changes (yes/no and free-text responses) and preferred alternate terminologies for low-risk PTCs (multiple choice and free-text responses). BN and CS collated and read through all of the questionnaire responses. Demographic responses, health literacy, ${ }^{27}$ cancer worry ${ }^{28}$ and participant's anxiety scores ${ }^{29}{ }^{30}$ have been included in table 1 , and the other responses were discussed and pooled with transcript data when analysing key overarching concepts.

\section{RESULTS}

\section{Participant characteristics}

Twenty-one men and twenty-six women of various ages from a range of socioeconomic backgrounds took part in six focus groups (table 1). Nineteen participants (40\%) had a university degree or above which is slightly higher than that of the general Australian population. ${ }^{31}$ Three participants $(6 \%)$ had experienced a personal diagnosis of cancer other than thyroid cancer and $29(62 \%)$ had an immediate family member with a diagnosis of cancer. Overall, the sample reported low levels of cancer worry and general anxiety.

The thematic analysis identified three main themes arising from the focus groups' discussions: (1) participants' initial perceptions and understanding of thyroid cancer, (2) reactions to thyroid cancer overdiagnosis and overtreatment information and (3) views and opinions about the strategy of changing the terminology. The latter two themes are further broken down into a number of subthemes which formed the majority of the 
Table 1 Participant characteristics $(n=47)$

\begin{tabular}{lc}
\hline Characteristic & Frequency $\mathbf{n}(\%)$ \\
\hline Age & \\
$18-34$ & $18(38.3)$ \\
$35-49$ & $11(23.4)$ \\
$50-64$ & $9(19.1)$ \\
$65+$ & $9(19.1)$ \\
\hline
\end{tabular}

Sex

\begin{tabular}{lc} 
Male & $21(44.7)$ \\
Female & $26(55.3)$ \\
Education & $19(40.4)$ \\
$\begin{array}{l}\text { University degree } \\
\text { Diploma, certificate or } \\
\text { apprenticeship }\end{array}$ & $19(40.4)$ \\
$\begin{array}{l}\text { Higher school certificate or leaving } \\
\text { certificate }\end{array}$ & $8(17.0)$ \\
No school or other qualifications & $1(2.1)$ \\
Marital Status & \\
Married/living with partner & $30(63.8)$ \\
Divorced/separated & $4(8.5)$ \\
Widowed & $2(4.3)$ \\
Single & $11(23.4)$ \\
Employment & \\
Full time & $22(46.8)$ \\
Part time & $11(23.4)$ \\
Retired & $9(19.1)$ \\
Studying & $1(2.1)$ \\
Other & $4(8.5)$ \\
Main language spoken at home & \\
English & $42(89.3)$ \\
Other & $5(10.7)$ \\
\hline Abigna/Tores Stat Isander &
\end{tabular}

Aboriginal/Torres Strait Islander

$\begin{array}{lc}\text { No } & 46(97.9) \\ \text { Aboriginal } & 1(2.1) \\ \text { Torres Strait Islander } & 0(0) \\ \text { Aboriginal and Torres Strait Islander } & 0(0) \\ \text { Health literacy* } & \\ \text { Adequate health literacy } & 44(93.6) \\ \text { Limited/marginal health literacy } & 3(6.4)\end{array}$

Personal cancer diagnosis $\uparrow$

\begin{tabular}{lc} 
No & $44(93.6)$ \\
\multicolumn{1}{l}{ Yes } & $3(6.4)$ \\
$\begin{array}{l}\text { Immediate family member diagnosed } \\
\text { with cancer }\end{array}$ & \\
$\quad$ No & $18(38.3)$ \\
Yes & $29(61.7)$ \\
Cancer worry & \\
$\quad$ Not worried at all & $14(29.8)$ \\
\hline
\end{tabular}

Continued
Table 1 Continued

\begin{tabular}{lc}
\hline Characteristic & Frequency $\mathbf{n}(\%)$ \\
\hline A bit worried & $25(53.2)$ \\
Quite worried or very worried & $7(14.9)$ \\
General anxiety (mean score)§ & 28.7 \\
\hline
\end{tabular}

*Single Item Literacy Screener. ${ }^{27}$

†Other than thyroid cancer.

$\ddagger A$ validated single item that measures level of worry about

developing cancer, using four response categories ranging from not worried at all to very worried. ${ }^{28}$ One response missing. $\S$ State trait anxiety inventory (short form), on a scale from 20 to 80 , with higher scores indicating greater levels of anxiety. ${ }^{29} 30$

discussion and data collected. Participants' quotations have been selected to demonstrate both common and diverse responses.

\section{Participants initial perceptions and understanding of thyroid cancer}

Across the focus groups most people had little knowledge of thyroid cancer which was evident in the number of general questions about thyroid cancer asked by participants in each group. While most had heard of thyroid cancer, the majority of participants did not know how it is detected, diagnosed and treated. Participants perceived thyroid cancer to be quite similar to other types of cancer in terms of the risk of the morbidity and mortality. Overall, participants believed that all cancers were harmful and deadly.

...with cancer we're constantly told like... it's going to get us, everyone... (FG3)

See like I think of cancer as like... you're very sick, like almost certain to be terminally ill. (FG4)

\section{Reactions to thyroid cancer overdiagnosis and overtreatment} information

Uncertainty about overdiagnosis and overtreatment

There was initially some overall scepticism about the concept of thyroid cancer overdiagnosis and overtreatment, and overdiagnosis and overtreatment information generally. This information was new to all participants, and seemed to cause some initial uncertainty and raise additional thoughts and questions. However, as each of the sessions progressed, there was a shift to more of a general acceptance and understanding of the information. Participant's questions and responses shifted and indicated to researchers that they were able to thoughtfully engage with the information presented. Alongside the acceptance and understanding of overdiagnosis and overtreatment came a sense of concern from participants that they had not been informed or educated about this type of information before. Participants expressed concern that this information was not public knowledge and queried whether this information was being discussed with current patients, as they felt it should be. Overall, there was a strong desire for more information 
and greater public and patient education about these topics in relation to low-risk thyroid cancer, as well as other low-risk cancers.

That doesn't make sense to me. What about those cancers that have been widely researched and tested. (FG1)

I wasn't aware of this, like, overdiagnosis and overtreatment. All I'm told is, you have to get checked and you have to do this. (FG3)

\section{Understanding and support for reducing overdiagnosis and overtreatment}

There were many participants who voiced their understanding and support for reducing overdiagnosis and overtreatment. Some felt that it was probably better not knowing that a low-risk cancer was present in the first place. If diagnosed themselves many expressed that they saw active surveillance as a good way to potentially reduce overtreatment. Some participants also felt it important that reducing overdiagnosis, and in particular overtreatment, would reduce financial costs for both patients and the healthcare system.

It just sounds like it's better not knowing. (FG5)

It's a cancer that you don't, you wouldn't even know that it was there. It's not affecting your life... if I personally got diagnosed and if it wasn't like a big medical thing and I didn't need the, um, surgery, then I probably wouldn't. (FG4)

Overdiagnosis and then overtreatment we're really creating a, a higher cost to the medical system for something that's just staying sort of baseline for most individuals. (FG6)

Concern about the implications of overdiagnosis and overtreatment A number of participants voiced concerns about the implications of thyroid cancer overdiagnosis and overtreatment. Their main concern was regarding the uncertainty of the risk of overdiagnosis and overtreatment. These participants were uncomfortable with the idea that at this time it is not known at an individual level who may be overdiagnosed and overtreated. Some participants were also concerned about the impact that this information may have on patient's quality of life. They felt that giving this information to someone and recommending active surveillance instead of treatment would be psychologically hard to deal with.

So you really don't know, or no one knows if overtreatment is actually happening? So like you're surmising that it is happening but no one knows yet. (FG2)

I guess it could be like a scary thing to live with also, just thinking, oh well something might have changed, um, maybe I do need to get surgery? Just constantly kind of thinking, oh what if. (FG4)
Views and opinions about the strategy of changing the terminology

The majority of participants were open to the idea of changing the terminology of low-risk thyroid cancers. However, some participants were quite unsure about how they felt about the idea, and a small group of participants remained strongly opposed throughout the discussions. Overall, participants understood and accepted the concept of the proposed strategy, although a few participants still struggled with how the strategy would ultimately work and what it would mean for them (as patients or community members).

I'm fine with it being changed. I think I'm actually for it ... but I do think you do need to be informed. (FG6)

...it's such a big shift in thinking and maybe, maybe that's what's making me feel uncomfortable. (FG1)

\section{Reasons for supporting changing the terminology}

The most dominant reason for participants supporting changes to cancer terminology was the psychological impact and stigma associated with the term 'cancer'. Overall, participants believed that the word 'cancer' was frightening and anxiety provoking, and suggested it may drive the preference for immediate treatment. Other ideas discussed included that the current definition of cancer may not actually represent the nature of this condition (as this type of thyroid cancer was lower risk than what they understood cancer to be), and that changing the terminology would help with the potential insurance difficulties current cancer patients may face.

As soon as you get that word 'cancer' it triggers, um, a reaction which is always negative. And it's like this perception that it's got to be treated. (FG3)

It's our association with the word cancer. So I think cancer, doom and gloom... But if there's such a lowrisk of someone dying from it, why phrase it and coin it cancer at all? (FG1)

\section{Reasons against changing the terminology}

The main reason participants expressed for being unsure about or strongly opposed to changing the terminology was the worry about the risk of progression. These participants did not feel comfortable with removing the term 'cancer' from diagnoses if there was a small risk that the tumour could progress and become more harmful. Participants also felt that changing the terminology would mask the truth of the diagnosis as it was technically still a cancer, and could cause confusion, particularly if patients searched the internet and discovered that their condition used to be referred to as cancer. Some participants were additionally worried that patients may not adhere to appropriate follow-up or management measures if the condition was not called cancer.

...what if I am part of that $3.8 \%$, where it has actually spread? That would not sit well with me. (FG3) 
Whether it be low-risk or high risk, it's still cancer. (FG6)

I think maybe changing those things might make me very gung ho and think, oh right, you know, I might not go for my next test. (FG2)

\section{Views and opinions about alternative terminology}

Participants had greatly varying opinions about terminology for low-risk thyroid cancers, and could not reach agreement on an alternate term. Some participants preferred the term 'abnormal cells', being familiar with this term's use for low-risk cervical lesions, while others disagreed, stating that 'abnormal' was equally anxiety provoking. Participants also voiced opinions on various possible terms to describe low-risk thyroid cancer (eg, preinvasive cancer, lump, lesion, nodule). A few participants suggested that rather than changing the terminology, some sort of risk stratification terminology could be used to describe the condition. Mainly participants wanted terminology that was clear, understandable (ie, did not include a lot of medical jargon) and would not cause confusion or further anxiety.

...it is abnormal but it's not introducing that, that fear factor word attached to it. (FG1)

Abnormal you can get some people anxious, 'cause it's like not normal... What's not normal about it? (FG3)

So I think you, you call it something like low risk cancer or something like that... Or something that still calls it a cancer but actually gives it an appropriate risk factor. (FG1)

\section{Other proposed strategies}

Aside from changes to terminology, participants felt strongly about two proposed strategies to reduce potential overdiagnosis and overtreatment of low-risk thyroid cancer. The first was the importance of medical professional involvement in informing and discussing these issues with patients (if they were not already doing so). The majority of participants felt that there was a need for clear explanations around the diagnosis of thyroid cancer (and cancer generally) and treatment options. The second strategy that was suggested was the need for better public education. Participants were unsure of why they had not heard about information on overdiagnosis and overtreatment of low-risk thyroid cancer or low-risk cancer generally before and suggested campaigns to promote greater public awareness.

It's very important for the doctor/patient relationship that if you are diagnosed with, with this, you know, as a result of something else, that information that, like you've shown us today, you've spoken to us today, is made very clear to the, to the patient. (FG3)

To me it's more about the education of what it means, rather than changing the name because it's scary. (FG2)
Maybe if the public was sort of educated in the same way you've just, you know, explained things to us... people would, um, make a more informed opinion about what they want to do. (FG3)

\section{DISCUSSION}

This study sheds light on public perceptions of information on thyroid cancer overdiagnosis and overtreatment, and potential strategies to help mitigate these in low-risk thyroid cancer and other similar conditions. Consistent with earlier studies, this community sample had a low pre-existing general awareness of overdiagnosis and overtreatment, ${ }^{32-37}$ including issues specifically related to thyroid cancer. The latter is not surprising as there is no formal screening programme in Australia, and testing does not receive the publicity that cancers like breast, prostate and lung cancer receive. There was initially scepticism among participants about the concept of overdiagnosis and overtreatment; however, as more information was provided, participants appeared to understand and accept the concepts and accept active surveillance as a possible alternative management strategy to mitigate overtreatment in low-risk thyroid cancer. These findings mirror other community-based studies that also indicate preferences towards active surveillance. ${ }^{16} 1738$ We observed, however, wide variation in views with some residual concerns about the evidence for overdiagnosis, and in particular, overtreatment of thyroid cancer.

Similar to the medical community, ${ }^{10}$ there is variation in the public's views about whether changing the terminology of low-risk thyroid cancer (and other low-risk cancers) is an adequate strategy to help reduce overdiagnosis and overtreatment. Our study participants were also uncertain about an appropriate and acceptable alternative term to replace 'cancer'. Reservations concerned worry about the risk of further progression of the disease and the need to still take the condition seriously, even if the terminology were changed. Concern about the risk of progression to invasive disease and the evidence of overdiagnosis and overtreatment is not surprising as it has been shown to be a factor driving clinician management recommendations. ${ }^{10}$ Furthermore, not being able to individually select who may be impacted was difficult for participants to comprehend and accept which has been voiced as a concern in understanding and communicating overdiagnosis information previously. ${ }^{32} 3335$

Notably, there was a strong overarching desire for greater patient and public education around overdiagnosis and overtreatment in cancer to complement any revisions to terminology. Participants wanted greater medical professional involvement in informing patients about relevant evidence and an increase in public health campaigns with information on these topics. Other studies have found that information on overdiagnosis and overtreatment is strongly desired by patients. ${ }^{37}$ In addition, studies demonstrate that those who should be the main source of providing such information to patients are physicians, 
Box 2 Summary of future implications for communicating about low-risk conditions

- Community members are able to understand the overarching concept of overdiagnosis and overtreatment when it is clearly presented and discussed.

- Providing the best available data on estimates of overdiagnosis and overtreatment may decrease public and patient uncertainty and make understanding more intuitive.

- The public generally views all cancers as being very harmful; therefore, widespread public conversation around cancer should shift to include information about the difference between lower risk cancers and higher risk cancers (eg, not all cancers are the same).

- Healthcare practitioners should use language which reduces patient anxiety and the strong preference for more invasive management options (eg, low risk).

- Where appropriate, healthcare practitioners should discuss concerns around overdiagnosis and overtreatment with patients including the option of less aggressive management options.

and often physicians themselves do not have adequate understanding or knowledge on the extent or harms of overdiagnosis. $^{39}{ }^{40}$ Although widespread implementation of this information may take time, strategies beyond changing disease terminology including mass media education, shared decision-making through patient decision aids and citizen juries have all also been suggested to be potentially effective for communicating about overdiagnosis or overtreatment. ${ }^{41}$ Box 2 summarises issues to consider when communicating to patients and the public about low-risk conditions.

Our study had both strengths and limitations. To our knowledge, this is the first study to investigate community perceptions of information about thyroid cancer overdiagnosis and overtreatment and community responses to the possibility of changing the name of low-risk PTCs. We had an adequate sample size for qualitative research which included participants of different age, gender and socioeconomic status. Participants were recruited via random digit dialling by an independent professional recruitment company.

The main limitation of our study was the amount and complexity of information that was presented to participants during the 2-hour focus group. Since participants were hearing this information for the first time in this short time period, participants may not have comprehensively understood each concept. We did not test participants' understanding; although our data indicated that most people engaged thoughtfully with the question of a terminology change and understood the information. Moreover, the qualitative nature of our study did not allow us to quantify the prevalence of public perceptions or preferences towards a change in terminology, rather it was used to explore a range of views and opinions which may lack generalisability. These views may have been influenced by the investigator's interactions during the presentation or by the views expressed by other participants. Furthermore, it can be argued that the thematic analysis may be prone to bias due to the dependence of the raters. However, this qualitative approach offers the advantage of potentially identifying themes or issues that may not have been apparent a priori. Additionally, to help ensure any bias related to presentation was minimised we arranged for it to be reviewed by two independent thyroid cancer clinicians (an international endocrinologist and a national thyroid surgeon). It was also piloted three times in order to ensure comprehensibility.

In conclusion, many community members in our study were open to a change in the terminology to describe low-risk cancer, but there were considerable variability in the public's willingness to accept this proposal. Participants, even those supportive of a change in terminology, were unable to propose an appropriate alternative terminology. Consequently, we conclude that some citizens might not accept a change in nomenclature to low-risk cancers, and could feel very unsure of or even resistant to changes in terminology. If such a change were to go ahead, careful consideration of how to inform both the public and current patients about the implications of the change, including changes to current patients' follow-up or treatments, would be needed. Involving community members and consumers in the renaming process could help ensure that any change to terminology will be understood and accepted by the public and patients alike. Moreover, there is a strong and apparently widely held desire for more information-to patients and to the publicon the topic of cancer overdiagnosis and overtreatment. Enhanced information provision would be essential to complement a change in terminology for PTC and other low-risk cancers.

\section{Author affiliations}

${ }^{1}$ Wiser Healthcare, School of Public Health, University of Sydney, Sydney, New South Wales, Australia

${ }^{2}$ Sydney Health Literacy Lab, School of Public Health, University of Sydney, Sydney, New South Wales, Australia

${ }^{3}$ Faculty of Medicine and Health, The University of Sydney, Sydney, Australia ${ }^{4}$ Centre for Research in Evidence-Based Practice, Bond University, Herston, Queensland, Australia

${ }^{5}$ Cancer Causes and Care, QIMR Berghofer Medical Research Institute, Herston, Queensland, Australia

${ }^{6}$ School of Public Health, The University of Queensland, Herston, Queensland, Australia

${ }^{7}$ Department of Endocrinology and Diabetes, Royal Brisbane and Women's Hospital, Herston, Queensland, Australia

${ }^{8}$ Division of Endocrinology, Diabetes, Metabolism and Nutrition, Mayo Clinic, Rochester, Minnesota, USA

Acknowledgements We thank Professor Michael Tuttle and Associate Professor Mark Sywak for their expertise in independently reviewing the focus group presentation, and Darlene Cox for her input and helpful comments as a consumer representative.

Contributors BN, RM, AB and KM conceived the study. BN, RM, AB, SJ, DM, JPB and $\mathrm{KM}$ were involved in designing the study and developing the methods. $\mathrm{BN}$ and CS coordinated the running of the study and conducted the focus groups. BN and CS analysed the data. BN drafted the manuscript. All authors contributed to the interpretation of the analysis, and critically revised and approved the manuscript.

Funding BN was supported by Sydney Catalyst Research Scholar Award. JPB was supported by the Karl-Erivan Haub Family Career Development Award in Cancer Research at Mayo Clinic in Rochester, honouring Richard F. Emslander, M.D. RM, 
SJ, DM and KM were supported by National Health and Medical Research Counci (NHMRC) fellowships (1124207, 1061341, 1092153, and 1029241, respectively). The project was supported by an NHMRC Centre of Research Excellence Grant (1104136).

Competing interests RM and KM report that they are co-organisers of the Preventing Overdiagnosis conferences, and the 2017 Australian National Summer on overdiagnosis. $A B$ reports she is chief investigator on a Australian National Health and Medical Research Council (NHMRC) Centre of Research Excellence Grant (1104136).

\section{Patient consent Obtained.}

Ethics approval The study was approved by the University of Sydney Human Research Ethics Committee (2017/813).

Provenance and peer review Not commissioned; externally peer reviewed.

Data sharing statement No additional data available.

Open access This is an open access article distributed in accordance with the Creative Commons Attribution Non Commercial (CC BY-NC 4.0) license, which permits others to distribute, remix, adapt, build upon this work non-commercially, and license their derivative works on different terms, provided the original work is properly cited, appropriate credit is given, any changes made indicated, and the use is non-commercial. See: http://creativecommons.org/licenses/by-nc/4.0/.

\section{REFERENCES}

1. Wiltshire JJ, Drake TM, Uttley L, et al. Systematic review of trends in the incidence rates of thyroid cancer. Thyroid 2016;26:1541-52.

2. Furuya-Kanamori L, Bell KJL, Clark J, et al. Prevalence of differentiated thyroid cancer in autopsy studies over six decades: a meta-analysis. J Clin Oncol 2016;34:3672-9.

3. Brito JP, Morris JC, Montori VM. Thyroid cancer: zealous imaging has increased detection and treatment of low risk tumours. BMJ 2013;347:f4706.

4. Davies L, Welch HG. Current thyroid cancer trends in the United States. JAMA Otolaryngol Head Neck Surg 2014;140:317-22.

5. Lee SH, Kim TY, Ryu J-S, et al. Trends analysis of characteristics of thyroid cancer patients in one medical center. Journal of Korean Endocrine Society 2008;23:35-43.

6. Lim H, Devesa SS, Sosa JA, et al. Trends in thyroid cancer incidence and mortality in the United States, 1974-2013. JAMA 2017;317:1338-48.

7. Miyauchi A, Ito $\mathrm{Y}$, Oda $\mathrm{H}$, et al. Insights into the management of papillary microcarcinoma of the thyroid. Thyroid 2018;28:23-31.

8. Hauch A, Al-Qurayshi Z, Randolph G, et al. Total thyroidectomy is associated with increased risk of complications for low- and highvolume surgeons. Ann Surg Oncol 2014;21:3844-52.

9. Lee DY, Seok J, Jeong WJ, et al. Prediction of thyroid hormone supplementation after thyroid lobectomy. J Surg Res 2015;193:273-8.

10. Nickel B, Brito JP, Barratt A, et al. Clinicians' views on management and terminology for papillary thyroid microcarcinoma: a qualitative study. Thyroid 2017;27:661-71.

11. Haugen BR, Alexander EK, Bible KC, et al. 2015 American thyroid association management guidelines for adult patients with thyroid nodules and differentiated thyroid cancer: the American thyroid association guidelines task force on thyroid nodules and differentiated thyroid cancer. Thyroid 2016;26:1-133.

12. Vaccarella S, Franceschi S, Bray F, et al. Worldwide Thyroid-Cancer Epidemic? The Increasing Impact of Overdiagnosis. N Engl J Med 2016;375:614-7.

13. Allegra CJ, Aberle DR, Ganschow P, et al. NIH state-of-the-science conference statement: diagnosis and management of ductal carcinoma in situ (DCIS). NIH Consens State Sci Statements 2009;26:1-27.

14. Esserman LJ, Thompson IM, Reid B, et al. Addressing overdiagnosis and overtreatment in cancer: a prescription for change. Lancet Oncol 2014;15:e234-42.

15. Omer ZB, Hwang ES, Esserman LJ, et al. Impact of ductal carcinoma in situ terminology on patient treatment preferences. JAMA Intern Med 2013:173:1830-1.
16. McCaffery K, Nickel B, Moynihan R, et al. How different terminology for ductal carcinoma in situ impacts women's concern and treatment preferences: a randomised comparison within a national community survey. BMJ Open 2015;5:e008094.

17. Nickel B, Barratt A, McGeechan K, et al. Effect of a change in papillary thyroid cancer terminology on anxiety levels and treatment preferences. JAMA Otolaryngol Head Neck Surg 2018;144:867.

18. Nickel B, Barratt A, Copp T, et al. Words do matter: a systematic review on how different terminology for the same condition influences management preferences. BMJ Open 2017;7:e014129.

19. Nikiforov YE, Seethala RR, Tallini G, et al. Nomenclature revision for encapsulated follicular variant of papillary thyroid carcinoma: a paradigm shift to reduce overtreatment of indolent tumors. JAMA Oncol 2016;2:1023-9.

20. Welch HG, Schwartz LM, Woloshin S. Overdiagnosed: making people sick in the pursuit of health. Boston: Beacon Press, 2011.

21. Bowen GA. Naturalistic inquiry and the saturation concept: a research note. Qualitative Research 2008;8:137-52.

22. Kuper A, Lingard L, Levinson W. Critically appraising qualitative research. BMJ 2008;337:a1035.

23. Hawley ST, Zikmund-Fisher B, Ubel P, et al. The impact of the format of graphical presentation on health-related knowledge and treatment choices. Patient Educ Couns 2008;73:448-55.

24. Ahn HS, Kim HJ, Welch HG. Korea's thyroid-cancer "epidemic"screening and overdiagnosis. N Engl J Med 2014;371:1765-7.

25. Kitzinger J. The methodology of Focus Groups: the importance of interaction between research participants. Sociology of Health and IIIness 1994;16:103-21.

26. Pope C. Qualitative research in health care: analysing qualitative data. BMJ 2000;320:114-6.

27. Morris NS, MacLean CD, Chew LD, et al. The single item literacy screener: evaluation of a brief instrument to identify limited reading ability. BMC Fam Pract 2006;7:21.

28. Hersch J, Barratt A, Jansen J, et al. Use of a decision aid including information on overdetection to support informed choice about breast cancer screening: a randomised controlled trial. Lancet 2015;385:1642-52.

29. Marteau TM, Bekker H. The development of a six-item short-form of the state scale of the Spielberger State-Trait Anxiety Inventory (STAI). Br J Clin Psychol 1992;31:301-6.

30. Spielberger CD. State-trait anxiety inventory for adults: sampler set. Palo Alto: Mind Garden, 1983.

31. Australian Bureau of Statistics. Education and work, Australia. 2016 http://www.abs.gov.au/ausstats

32. Hersch J, Jansen J, Barratt A, et al. Women's views on overdiagnosis in breast cancer screening: a qualitative study. BMJ 2013;346:f158.

33. Waller J, Douglas E, Whitaker KL, et al. Women's responses to information about overdiagnosis in the UK breast cancer screening programme: a qualitative study. BMJ Open 2013;3:e002703.

34. Moynihan R, Nickel B, Hersch J, et al. Public opinions about overdiagnosis: a national community survey. PLoS One 2015;10:e0125165

35. Park SH, Lee B, Lee S, et al. A qualitative study of women's views on overdiagnosis and screening for thyroid cancer in Korea. BMC Cancer 2015;15:858

36. Lee S, Lee YY, Yoon HJ, et al. Responses to overdiagnosis in thyroid cancer screening among Korean women. Cancer Res Treat 2016;48:883-91.

37. Wegwarth O, Gigerenzer G. Less is more: overdiagnosis and overtreatment: evaluation of what physicians tell their patients about screening harms. JAMA Intern Med 2013;173:2086-7.

38. Nickel B, Barratt A, Hersch J, et al. How different terminology for ductal carcinoma in situ (DCIS) impacts women's concern and management preferences: A qualitative study. Breast 2015;24:673-9.

39. Wegwarth O, Schwartz LM, Woloshin S, et al. Do physicians understand cancer screening statistics? A national survey of primary care physicians in the United States. Ann Intern Med 2012;156:340-9.

40. Hoffmann TC, Del Mar C. Clinicians' expectations of the benefits and harms of treatments, screening, and tests: a systematic review. JAMA Intern Med 2017;177:407-19.

41. McCaffery KJ, Jansen J, Scherer LD, et al. Walking the tightrope: communicating overdiagnosis in modern healthcare. BMJ 2016;352:i348. 